\title{
EST analysis of the scaly green flagellate Mesostigma viride (Streptophyta): Implications for the evolution of green plants (Viridiplantae)
}

\author{
Andreas Simon ${ }^{\dagger 1}$, Gernot Glöckner ${ }^{\dagger 2}$, Marius Felder ${ }^{2}$, Michael Melkonian ${ }^{1}$ \\ and Burkhard Becker*1
}

Address: ${ }^{1}$ Botanical Institute, University of Cologne, Gyrhofstr. 15, 50931 Cologne, Germany and ${ }^{2}$ Genome Analysis, Leibniz Institute for Age Research - Fritz Lipmann Institute, Beutenbergstr. 11, 07745 Jena, Germany

Email: Andreas Simon - andreas.simon@uni-koeln.de; Gernot Glöckner - gernot@imb-jena.de; Marius Felder - mfelder@imb-jena.de; Michael Melkonian - michael.melkonian@uni-koeln.de; Burkhard Becker* - b.becker@uni-koeln.de

* Corresponding author †Equal contributors

Published: I3 February 2006

BMC Plant Biology2006, 6:2 doi:10.1 I86/147I-2229-6-2

This article is available from: http://www.biomedcentral.com/I47/-2229/6/2

(C) 2006Simon et al; licensee BioMed Central Ltd.

This is an Open Access article distributed under the terms of the Creative Commons Attribution License (http://creativecommons.org/licenses/by/2.0), which permits unrestricted use, distribution, and reproduction in any medium, provided the original work is properly cited.

\begin{abstract}
Background: The Viridiplantae (land plants and green algae) consist of two monophyletic lineages, the Chlorophyta and the Streptophyta. The Streptophyta include all embryophytes and a small but diverse group of freshwater algae traditionally known as the Charophyceae (e.g. Charales, Coleochaete and the Zygnematales). The only flagellate currently included in the Streptophyta is Mesostigma viride Lauterborn. To gain insight into the genome evolution in streptophytes, we have sequenced 10,395 ESTs from Mesostigma representing 3,300 independent contigs and compared the ESTs of Mesostigma with available plant genomes (Arabidopsis, Oryza, Chlamydomonas), with ESTs from the bryophyte Physcomitrella, the genome of the rhodophyte Cyanidioschyzon, the ESTs from the rhodophyte Porphyra, and the genome of the diatom Thalassiosira.
\end{abstract}

Results: The number of expressed genes shared by Mesostigma with the embryophytes ( $90.3 \%$ of the expressed genes showing similarity to known proteins) is higher than with Chlamydomonas (76.I $\%)$. In general, cytosolic metabolic pathways, and proteins involved in vesicular transport, transcription, regulation, DNA-structure and replication, cell cycle control, and RNA-metabolism are more conserved between Mesostigma and the embryophytes than between Mesostigma and Chlamydomonas. However, plastidic and mitochondrial metabolic pathways, cytoskeletal proteins and proteins involved in protein folding are more conserved between Mesostigma and Chlamydomonas than between Mesostigma and the embryophytes.

Conclusion: Our EST-analysis of Mesostigma supports the notion that this organism should be a suitable unicellular model for the last flagellate common ancestor of the streptophytes. Mesostigma shares more genes with the embryophytes than with the chlorophyte Chlamydomonas reinhardtii, although both organisms are flagellate unicells. Thus, it seems likely that several major physiological changes (e.g. in the regulation of photosynthesis and photorespiration) took place early during the evolution of streptophytes, i.e. before the transition to land. 


\section{Background}

The Viridiplantae (literally meaning green plants) include all green algae and embryophyte plants. They represent a monophyletic group of organisms, which display a surprising diversity with respect to their morphology, cell architecture, life histories and reproduction, and their biochemistry. The colonization of the terrestrial habitat by streptophyte algae 450 - 470 million years ago [reviewed in [1]] was undoubtedly one of the most important steps in the evolution of life on earth [2-4], which paved the way for the evolution of the various groups of land plants (embryophytes = bryophytes, pteridophytes and spermatophytes) resulting in our current terrestrial ecosystems [5].

A thorough understanding of the evolution of land plants requires knowledge about the phylogeny of green algae and embryophytes as well as insight into the evolution of plant genomes with special reference to developmental processes. Whereas our knowledge about the phylogeny of the Viridiplantae has greatly increased over the last years, the latter has hardly been addressed to date.

The Viridiplantae are grouped into two divisions: the Chlorophyta and the Streptophyta [6]. The Chlorophyta comprise the vast majority of green algae including most scaly green flagellates (e.g. Pyramimonas, Tetraselmis), the Ulvophyceae (e.g. Ulva, Acetabularia), Chlorophyceae (e.g. Chlamydomonas, Volvox) and Trebouxiophyceae (e.g. Chlorella) $[7,8]$. The Streptophyta include all embryophyte plants and a diverse paraphyletic assemblage of freshwater green algae, the Charales (stoneworts), Coleochaete, the Zygnematophyceae and a few other taxa [9]. Currently, the Charales are thought to be the sister group of the embryophytes suggesting that the evolution of true land plants already started with a complex organism [10]. Remarkably, only a single scaly green flagellate Mesostigma viride Lauterborn, has been found to belong to the Streptophyta [10-13]. The exact phylogenetic position of Mesostigma viride, however, is still controversial [10-12,1416]. Mesostigma has recently attracted much attention as a putative key organism for the understanding of the early evolution of the Streptophyta [17-20].
Two aspects in the evolution of land plants seem to be important in this respect. First, many key evolutionary inventions of plants took already place within the streptophyte algae. According to Graham et al. [21] one can distinguish several major transitions in the evolution of land plants starting with a Mesostigma-like flagellate ancestor: development of a cellulosic cell wall, multicellularity, cytokinesis by a phragmoplast, plasmodesmata, apical meristematic cell and apical cell proliferation leading to branching, asymmetric cell division, cell differentiation, retention of zygotes, heteromorphic life history, and a root meristem. Of these distinguishing features only the latter two evolved not until the embryophytes emerged. Second, the colonization of the terrestrial habitat with its exposure to air, increased solar radiation and life in a desiccating environment led to adaptations of cell architecture, metabolism and body plan to survive in the terrestrial ecosystems [5]. The evolutionary history of these adaptations is currently not known. Important questions are: How did the green algal progenitor adapt to the terrestrial habitat? Which genomic changes were associated with this transition? And which of these genes are derived from streptophyte green algae? To gain insight into these questions we have started to analyze ESTs from various streptophyte algal lineages.

Here, we present an analysis of 10,395 ESTs representing 3306 non-redundant expressed genes obtained from Mesostigma viride. We show that the number of genes shared is higher between Mesostigma and the embryophytes than between Mesostigma and Chlamydomonas. Comparison of expressed genes from Mesostigma with the genomes of Arabidopsis, Chlamydomonas, the red alga Cyanidioschyzon, and rice as well as ESTs from Physcomitrella and Porphyra allowed us to identify conserved and derived cellular functions within the different evolutionary lines and to obtain a first insight into the metabolic capabilities of the flagellate ancestor of green plants.

\section{Results}

Preparation and characterization of libraries

Total RNA was isolated from an axenic culture of Mesostigma viride during the light phase. The culture con-

Table I: Mesostigma viride cDNA libraries used.

\begin{tabular}{|c|c|c|c|c|c|c|}
\hline Name & cDNA & $\begin{array}{l}\text { Number of primary } \\
\text { clones }\end{array}$ & $\begin{array}{c}\text { Percentage } \\
\text { recombinant clones }\end{array}$ & size of inserts' (bp) & $\begin{array}{l}\text { average size of } \\
\text { inserts' (bp) }\end{array}$ & $\begin{array}{c}\text { number of ESTs } \\
\text { sequenced }\end{array}$ \\
\hline Mesol & small size fraction & 310000 & 90 & $250-2000$ & 706 & 100 \\
\hline Meso2 & large size fraction & 295000 & 88 & $600-3200$ & 1142 & 4954 \\
\hline Meso3 & $\begin{array}{l}\text { total cDNA } \\
\text { normalized }\end{array}$ & 106000 & 63 & $100-1100$ & 650 & 535 \\
\hline Meso4 & $\begin{array}{l}\text { large size fraction } \\
\text { normalized }\end{array}$ & 304000 & 56 & $400-6600$ & 2025 & $\begin{array}{c}2403 \mathrm{cDNAs}^{\mathrm{cDNA}}=4806 \\
\text { ESTs }^{2}\end{array}$ \\
\hline
\end{tabular}

\footnotetext{
1 determined by agarose gel electrophoresis, ${ }^{2}$ sequenced from 3 ' and 5 'end
} 
Table 2: Summary of Mesostigma viride expressed genes obtained from four cDNA libraries (Meso I - Meso 4).

\begin{tabular}{|c|c|c|c|}
\hline Category & & $\begin{array}{l}\text { No of } \\
\text { contigs }\end{array}$ & \\
\hline Mitochondrial' & & 36 & \\
\hline Plastidicl & & 65 & \\
\hline Bacterial $^{2}$ & & 193 & \\
\hline Novel & & $169 \mid$ & \\
\hline & $\begin{array}{l}\text { with recognizable protein motif } \\
\text { no protein motifs }\end{array}$ & & $\begin{array}{l}574 \\
1117\end{array}$ \\
\hline Similarity & & 1315 & \\
\hline & with known function ${ }^{3}$ & & 574 \\
\hline & unknown function ${ }^{4}$ & & 395 \\
\hline & low similarity 5 & & 346 \\
\hline Total Contigs & & 3300 & \\
\hline
\end{tabular}

I sequences showing only similarity to organelle genomes. ${ }^{2}$ sequences showing only similarity to bacterial sequences or the highest similarity to bacterial sequences; the origin of these putative bacterial contaminations is currently not clear, as bacteria-free cultures of Mesostigma were used. ${ }^{3}$ similarity to proteins with a well-defined function (BLAST score $>100$ ). ${ }^{4}$ similarity to conserved proteins with no established function (BLAST score $>100$ ). ${ }^{5}$ low similarity to proteins from a few organisms (BLAST score generally between 100 and 200); might reflect conserved protein domains.

tained about $5 \%$ cell division stages. The isolated RNA was used for the construction of 4 different cDNA libraries (Meso 1 - Meso 4). Meso 1 and 2 differed in the size of the cloned inserts. For Meso 3 and 4 full-length enriched cDNA was prepared and normalized prior to cloning. Meso 3 was obtained from the total normalized fulllength enriched cDNA, whereas for Meso 4 the normalized full-length enriched cDNA was size-fractionated by gel permeation chromatography to remove small fragments. The basic characteristics of the four libraries are given in Table 1.

Initially, about 100-500 ESTs were sequenced from all libraries and analyzed by BLASTX against the Swissprot and translated Genbank databases. Since the Meso 2 and 4 libraries containing the larger inserts gave more promising results, we subsequently sequenced about 4000 additional ESTs from the Meso 2 and Meso 4 libraries, respectively yielding a total of 10,395 reads $(5,527,413$ bp). Based on comparison with published sequences from Mesostigma viride the rate of sequencing error was determined to be generally between $1 \%$ and $7 \%$ (average $4 \%$ ) depending on the quality of the sequence.

ESTs were assembled using the PHRAP software yielding 3300 contigs with an average size of 769 bases $(57-4452$ bases) after manual curation. Further analysis based on sequence similarity searches revealed that 294 of these contigs were of plastidic, mitochondrial, or possibly bacterial origin (sequences showing the highest similarity to organellar or bacterial genomes, Table 2). These contigs
Table 3: Functional classification of $\mathbf{3 0 0 6}$ Mesostigma viride contigs using the KOG system [43] and an expectation threshold of $\mathrm{e}=10^{-7}$.

\begin{tabular}{|c|c|}
\hline Functional Category & $\begin{array}{l}\text { No. of } \\
\text { Contigs }\end{array}$ \\
\hline INFORMATION STORAGE AND PROCESSING & 236 \\
\hline [J] Translation, ribosomal structure and biogenesis & 168 \\
\hline [A] RNA processing and modification & 28 \\
\hline [K] Transcription & 26 \\
\hline [L] Replication, recombination and repair & 9 \\
\hline [B] Chromatin structure and dynamics & 5 \\
\hline CELLULAR PROCESSES AND SIGNALING & 209 \\
\hline $\begin{array}{l}\text { [D] Cell cycle control, cell division, chromosome } \\
\text { partitioning }\end{array}$ & 7 \\
\hline [Y] Nuclear structure & 0 \\
\hline [V] Defense mechanisms & 2 \\
\hline [T] Signal transduction mechanisms & 31 \\
\hline [M] Cell wall/membrane/envelope biogenesis & 9 \\
\hline$[N+Z]$ Cytoskeleton and cell motility & 18 \\
\hline [W] Extracellular structures & 0 \\
\hline $\begin{array}{l}{[\mathrm{U}] \text { Intracellular trafficking, secretion, and vesicular }} \\
\text { transport }\end{array}$ & 41 \\
\hline $\begin{array}{l}\text { [O] Posttranslational modification, protein turnover, } \\
\text { chaperones }\end{array}$ & 101 \\
\hline METABOLISM & 212 \\
\hline [C] Energy production and conversion & 87 \\
\hline [G] Carbohydrate transport and metabolism & 35 \\
\hline [E] Amino acid transport and metabolism & 24 \\
\hline [F] Nucleotide transport and metabolism & 9 \\
\hline$[\mathrm{H}]$ Coenzyme transport and metabolism & 13 \\
\hline [l] Lipid transport and metabolism & 21 \\
\hline [P] Inorganic ion transport and metabolism & 16 \\
\hline $\begin{array}{l}\text { [Q] Secondary metabolites biosynthesis, transport and } \\
\text { catabolism }\end{array}$ & 7 \\
\hline POORLY CHARACTERIZED & 158 \\
\hline$[R]$ General function prediction only & 55 \\
\hline [S] Function unknown & 37 \\
\hline$[X]$ Unnamed protein & 66 \\
\hline Unknown & 2191 \\
\hline
\end{tabular}

were excluded from the data set. 1315 of the 3006 contigs analyzed (44\%) showed significant similarity at the protein level to sequences from the public databases (Table 2 ). Hence, approximately $56 \%$ of the contigs represent either novel sequences with unknown function or untranslated regions of a gene. However, when the 1691 contigs with no significant similarity to known proteins were searched against the Interpro protein motif database, $574(33.9 \%)$ of these contigs contained a recognizable protein motif (Table 2 ). The most common protein motifs found in all 3006 expressed gene sequences were bipartite nuclear localization signals (IPR001472, 197x), prolinerich regions (IPR000694, 150x) and cytochrome c hemebinding sites (IPR000345, 99x). 


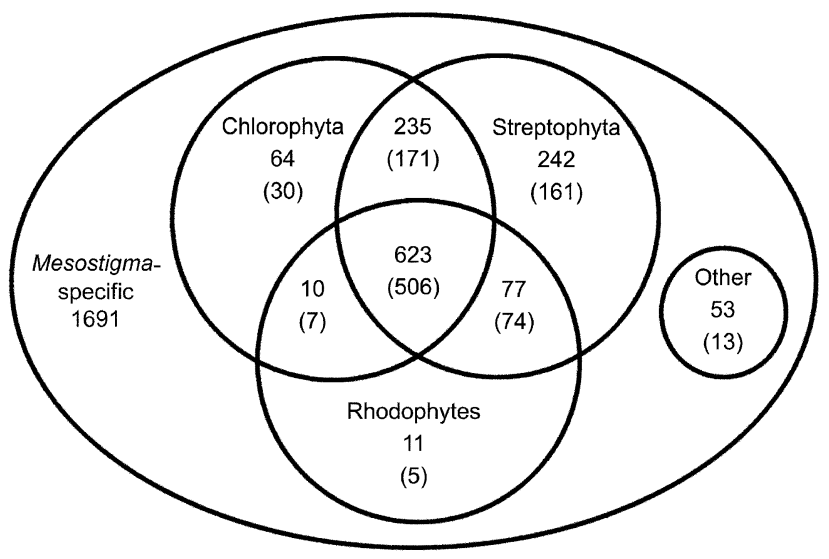

Figure I

Classification of expressed genes from Mesostigma according to the presence of similar proteins in other organisms in a Venn diagram. All non-redundant expressed genes were used as a query in (t)blastx similarity searches with the Swissprot, Genbank, Chlamydomonas, Cyanidioschyzon, Porphyra, Physcomitrella, Arabidopsis and Oryza data sets. The outermost circle represents all Mesostigma expressed genes. The inner circles, which are labeled chlorophyte, streptophyte and rhodophyte, represent genes, which have similarity to chlorophyte, streptophyte or rhodophyte sequences, respectively. The areas depicted are not proportional to the gene numbers and the number of Mesostigma expressed genes in each category is written in each segment. Numbers in brackets indicate the number of expressed genes in a category after removal of low similarity hits (see Table 2 for a definition of low similarity hits).

A functional catalogue was assembled using the 3006 Mesostigma contigs and the KOG-database and is presented in Table 3. As expected for an interphase cell, genes in the categories (1) translation, ribosomal structure and biogenesis (168), (2) posttranslational modification, protein turnover, chaperones (101), and (3) energy production and conversion (87) are represented by the largest number of contigs (Table 3 ). In the following, the assembled contigs are referred to as (expressed) genes.

\section{Classification of Mesostigma ESTs according to homologous genes in other organisms}

EST data represent only a fraction of all genes of an organism. Thus, comparisons of EST data alone cannot be used to describe unique or shared genes of an organism. For embryophytes, chlorophytes and red algae complete genome sequences of at least one organism exist. This makes it possible to find potential orthologous genes if present. Moreover, the surplus of genes of an organism in respect to a complete genome can be detected in EST data. In tBLASTX analyses of the 1315 expressed genes with similarity to known proteins $90.3 \%$ matched proteins from streptophytes, $76.1 \%$ from chlorophytes and $61 \%$ from rhodophytes, respectively. In addition, 46 genes showed similarity to known proteins, which have not been reported from plants or red algae to date. The overlap of Mesostigma genes with different organisms can be visualized in a Venn diagram (Figure 1). For 211 genes, we detected similar proteins only within the streptophyte but not in the chlorophyte or rhodophyte lineages. Conversely, for 62 genes we detected similar proteins only within the chlorophyte but not in the streptophyte or rhodophyte lineages. Surprisingly, we also found 6 genes which showed significant similarity to rhodophyte proteins but for which we could not detect any similar protein sequences within the Viridiplantae. Removal of BLAST hits with significant but low similarity (see Table 2) reduced the overall numbers to 972 expressed genes, but gave similar results (Figure 1). A complete list of genes showing only similarity to proteins with known functions present in specific subgroups of organisms can be found in supplemental Table 1 [see Additional file 1]. We will discuss important differences below.

\section{Overall protein similarities between various photoautotrophic organisms}

To compare the overall similarity between Mesostigma and various photoautotrophic organisms with completed genomes or large data sets of ESTs, we decided to calculate the average identity of a protein between Mesostigma and the various organisms. To compare Mesostigma genes with the genomes or ESTs from different organisms, we calculated the average identity (AI) between Mesostigma and another organism as the mean value of all pair wise identities of the BLAST-matches for each organism (Table 4).

The AI between Mesostigma and Chlamydomonas or the embryophytes are very similar. The highest AI value obtained was for Physcomitrella/Mesostigma followed by Arabidopsis/Mesostigma, Chlamydomonas/ Mesostigma and Oryza/Mesostigma. The full data set includes many proteins, which we detected only in some species using Mesostigma expressed genes as a query. Therefore, we constructed a constrained data set (314 expressed genes, including at least 46 nuclear encoded plastidic, 9 nuclear encoded mitochondrial, and 73 cytosolic ribosomal proteins), containing only Mesostigma genes which gave matches with all completed genomes from photoautotrophic eukaryotic organisms (including the diatom Thalassiosira). This constrained data set represents a conserved core set of nuclear encoded expressed proteins from photoautotrophic eukaryote organisms. We calculated AI values for the constrained data set using complete genomes and the available ESTs of Physcomitrella, Porphyra, and Chlamydomonas. The results are included in Table 4. We obtained the highest AI-values in the constrained data set for the three embryophytes, followed by Chlamydomonas. The similar AI values for the three differ- 
Table 4: Comparison of the Mesostigma expressed genes with the genomes and ESTs from various organisms. Average identity (AI) of pair wise comparisons of Mesostigma expressed genes with the indicated organismal data set.

\begin{tabular}{|c|c|c|c|c|c|c|c|c|}
\hline \multirow{2}{*}{$\begin{array}{l}\text { Data set } \\
\text { (No. of contigs) }\end{array}$} & \multicolumn{2}{|c|}{ Chlamydomonas } & \multirow{2}{*}{$\begin{array}{c}\text { Cyanidioschyzon } \\
\text { Genome }\end{array}$} & \multirow{2}{*}{$\begin{array}{c}\text { Porphyra } \\
\text { EST }\end{array}$} & \multirow{2}{*}{$\begin{array}{c}\text { Thalassiosira } \\
\text { Genome }\end{array}$} & \multirow{2}{*}{$\begin{array}{c}\text { Physcomitrella } \\
\text { EST }\end{array}$} & \multirow{2}{*}{$\begin{array}{c}\text { Arabidopsis } \\
\text { Genome }\end{array}$} & \multirow{2}{*}{$\begin{array}{c}\text { Oryza } \\
\text { Genome }\end{array}$} \\
\hline & Genome & EST & & & & & & \\
\hline All (969) & 0.573 & 0.563 & 0.522 & 0.528 & 0.528 & 0.590 & 0.577 & 0.565 \\
\hline Constrained (3|4) & 0.648 & $0.653(n=244)^{1)}$ & 0.557 & $\left.0.585(n=188)^{1}\right)$ & 0.569 & $0.675(n=301)^{1)}$ & 0.679 & 0.671 \\
\hline $\begin{array}{l}\text { Evolutionary } \\
\text { distance } D^{2)}\end{array}$ & 0.473 & 0.463 & 0.658 & 0.597 & 0.631 & 0.425 & 0.418 & 0.432 \\
\hline
\end{tabular}

The total data set contains all Mesostigma expressed genes with significant similarity to proteins from other organisms with known or unknown function (see Table 2). The constrained data set contains only Mesostigma expressed genes with significant similarity to proteins in all completely sequenced eukaryotic autotroph organisms.

I) Number of ESTs showing similarity to Mesostigma expressed genes from the constrained data set in a tBLASTX analysis. ${ }^{2)}$ Evolutionary distances were calculated using the constrained data set and the approximation given by Kimura [28]: $D=-\ln \left(I-p-0.2 p^{2}\right)$, where $p$ is the fraction of amino acid that differs between the two species.

ent embryophytes suggest that the overall evolutionary rate was very similar for the embryophytes investigated, when compared with Mesostigma (see below).

To test whether the observed differences are significant a paired students t-test was performed, and the results are shown in Table 5. Applying a significance level of 0.0072 [0.05/7 Bonferroni adjustment [22]] the differences in AI between Mesostigma/Chlamydomonas and Mesostigma/ embryophytes are highly significant (Table 5), whereas the differences in AI among the embryophytes are not significant (Table 5). Furthermore, when we varied the numbers of expressed genes used for the calculation of the AI, we observed that when more than 100 ESTs were included the significance of the differences became very stable (Fig. 2A). In addition, to evaluate the consistency of the data set we calculated 8 times the AI for 150 randomly selected expressed genes from the constrained data set. A clear difference between the AI from the various organisms was always observed (Fig. 2B $1-8$ ). The expression level of the expressed genes (as revealed by the number ESTs in a contig) had no effect on the differences between the investigated organisms (Fig. 2B, compare 9 and 10), although highly expressed genes are better conserved (Fig. 2B, 9 and 10).

Two other results are remarkable. First, for the calculation of the AI it is possible to use large EST-data sets instead of genomes. We obtained the same result for Mesostigma/ Chlamydomonas genome and for Mesostigmal Chlamydomonas ESTs (AI $=0.653$ for both data sets; $\mathrm{p}=$ 0,975, Table 5, using 244 expressed genes from Mesostigma). Similarly, when Mesostigma/Physcomitrella ESTs were compared with the Mesostigma/Arabidopsis genome

Table 5: Statistical significance of the obtained Al values. A paired students t-test was performed for the constrained data set to test whether the observed differences between the average identity of pair wise comparisons of Mesostigma expressed genes with the indicated organismal data set are significant. Differences are considered significant when $p$ is $<0.007$ I ( $0.05 / 8$ Bonferroni adjustment [22]).

\begin{tabular}{|c|c|c|c|c|c|c|c|}
\hline & Variablel & $\begin{array}{l}\text { No. of genes } \\
\text { shared }^{2}\end{array}$ & mean $^{3}$ & $\begin{array}{l}\text { standard } \\
\text { deviation }\end{array}$ & t-value & $\begin{array}{l}\text { Degrees of } \\
\text { freedom }\end{array}$ & $\rho$ \\
\hline I & $\begin{array}{l}\text { Mesostigma/Chlamydomonas } \mathrm{G} \\
\text { Mesostigma/Chlamydomonas } \mathrm{E}\end{array}$ & 244 & $\begin{array}{l}0.652992 \\
0.652992\end{array}$ & $\begin{array}{l}0.149868 \\
0.151255\end{array}$ & $-0,03107$ & 243 & 0.975239 \\
\hline 2 & $\begin{array}{l}\text { Mesostigma/Chlamydomonas G } \\
\text { Mesostigma/Physcomitrella } E\end{array}$ & 301 & $\begin{array}{l}0.649934 \\
0.674618\end{array}$ & $\begin{array}{l}0.149292 \\
0.153641\end{array}$ & -3.24578 & 300 & 0.001304 \\
\hline 3 & $\begin{array}{l}\text { Mesostigma/Chlamydomonas G } \\
\text { Mesostigma/Arabidopsis G }\end{array}$ & 314 & $\begin{array}{l}0.648057 \\
0.677994\end{array}$ & $\begin{array}{l}0.148512 \\
0.140940\end{array}$ & -4.44025 & 313 & 0.000012 \\
\hline 4 & $\begin{array}{l}\text { Mesostigma/Chlamydomonas G } \\
\text { Mesostigma./Oryza G }\end{array}$ & 314 & $\begin{array}{l}0.648057 \\
0.670382\end{array}$ & $\begin{array}{l}0.148512 \\
0.148384\end{array}$ & $-3.1537 \mid$ & 313 & 0.001768 \\
\hline 5 & $\begin{array}{l}\text { Mesostigma/Physcomitrella } E \\
\text { Mesostigma/Arabidopsis G }\end{array}$ & 302 & $\begin{array}{l}0.675364 \\
0.681159\end{array}$ & $\begin{array}{l}0.153933 \\
0.140567\end{array}$ & -135783 & 301 & 0.175535 \\
\hline 6 & $\begin{array}{l}\text { Mesostigma/Physcomitrella } E \\
\text { Mesostigma./Oryza G }\end{array}$ & 302 & $\begin{array}{l}0.675364 \\
0.673311\end{array}$ & $\begin{array}{l}0.153933 \\
0.147624\end{array}$ & 0,43158 & 301 & 0.666355 \\
\hline 7 & $\begin{array}{l}\text { Mesostigma/Arabidopsis G Mesostigma./ } \\
\text { Oryza G }\end{array}$ & 314 & $\begin{array}{l}0.678730 \\
0.671175\end{array}$ & $\begin{array}{l}0.141321 \\
0.148813\end{array}$ & 2.480053 & 313 & 0.013660 \\
\hline
\end{tabular}

1 Compared data sets $\mathrm{E}=\mathrm{EST}, \mathrm{G}=$ Genome. ${ }^{2} \mathrm{No}$. of genes shared between the compared data sets. ${ }^{3} \mathrm{Al}$ recalculated on the basis of the genes shared between the compared datasets. ${ }^{4}$ Standard deviation of ${ }^{3}$. 


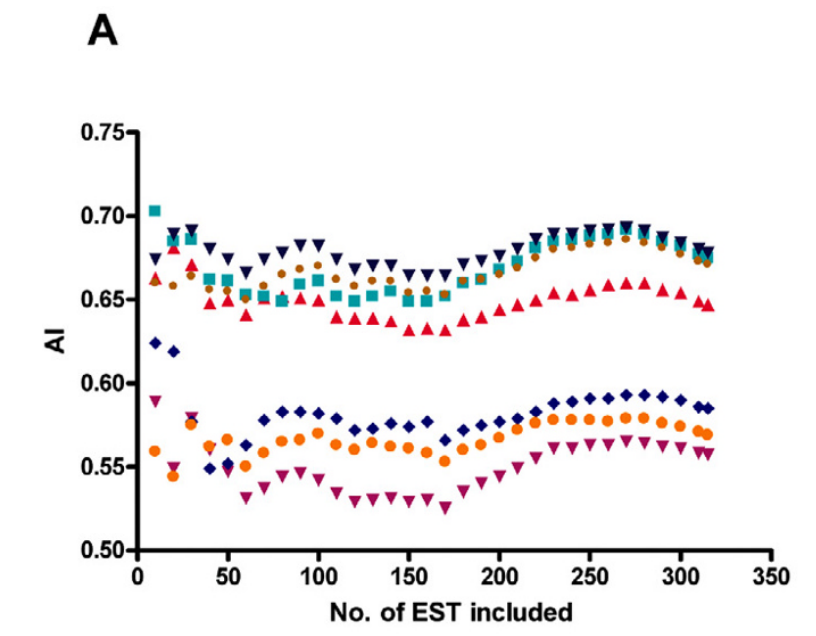

B

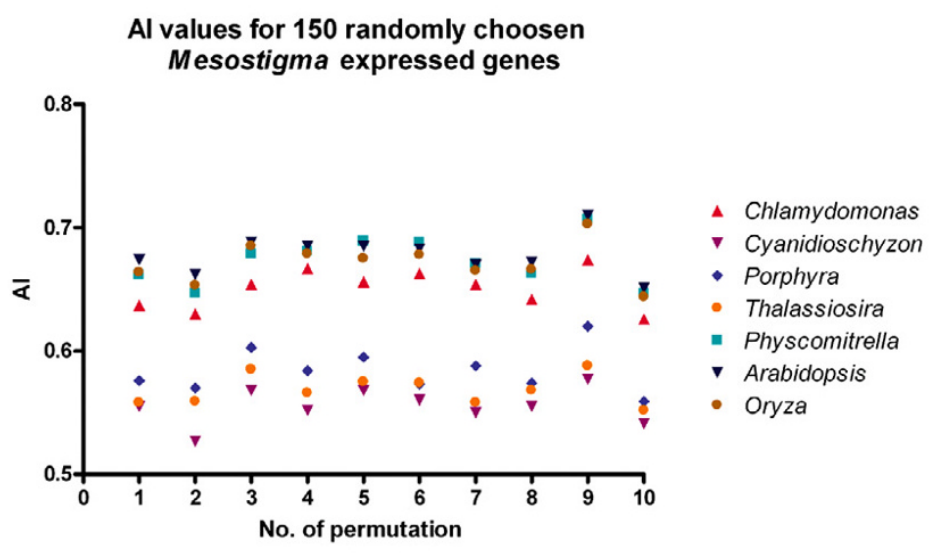

Figure 2

Consistency of the constrained data set used to calculate Al values. (A) The figure illustrates the effect of the number of genes included in the Al-values. The significant differences in the Al values are stable when more than I50 genes are included. (B) 150 genes were resampled randomly and the Als calculated for the indicated organisms (I - 8). Al values were calculated for the 150 most strongly ( 9 , as revealed by the number of ESTs in a contig) and weakly (I0, only single ESTs) expressed genes.

and with the Mesostigma/Oryza genome only small differences were observed $(\mathrm{AI}=0,675 / 0,681 ; 0,675 / 0,673$ respectively, using 302 expressed genes from Mesostigma, Table 5). Statistical analysis (paired students t-test) showed that the observed differences are not significant. Furthermore, we note that the genome of the diatom Thalassiosira pseudonana shows a similar AI in respect to Mesostigma as the red algal genome and ESTs (Table 4). The difference values of these distantly related genomes represent presumably an upper threshold for reasonable AI value calculations.

\section{Analysis of metabolic pathways}

ESTs have been widely used for the identification of metabolic pathways [23]. A complete list of all metabolic pathways identified is presented in supplemental Table 2 [see Additional file 2]. Indeed, many ESTs showed similarity to proteins required for photosynthesis (66 expressed genes), nucleotide synthesis (6), nucleotide sugar conversion, the biosynthesis of precursors of scale polysaccharides (6), heme and chlorophyll biosynthesis (6), fatty acid and lipid biosynthesis (9), terpenoid biosynthesis (6), glycolysis (11) and the TCA-cycle including pyruvate dehydrogenase and respiration (12). The biosynthetic pathways for several amino acids were also well represented in our ESTs (21 expressed genes for Ala, Arg, Gly, Ile, Leu, Lys, Pro, Ser, Thr, Trp and Val). However, for several other amino acids (Asn, Asp, Cys, Gln, Glu, His, Met, Phe, Tyr) we did not find a single EST which could be matched to the known biosynthetic pathways.
All enzymes except one (triose isomerase) of the Calvin cycle are represented by at least one EST. Interestingly, we found several genes coding for subunits of the plastidic GAPDH. In angiosperms the plastidic GAPDH consists of an $\mathrm{A}_{2} \mathrm{~B}_{2}$ heterotetramer [24]. Compared to GAPDH A, which is present in the plastids of all eukaryotic algae, GAPDH B has a C-terminal extension that contains the two conserved cysteine residues, which are required for regulation by the thioredoxin system. To our knowledge, GAPDH B has only been reported from streptophytes. Two genes of Mesostigma showed significant similarity to GAPDH B from angiosperms. We present an alignment of the C-terminus of Mesostigma GAPDH B with the C-terminus of spinach GAPDH B in Figure 3. The two sequences are very similar and the two cysteines required for regulation by the thioredoxin system are conserved in Mesostigma indicating that the activity of plastidic GAPDH came under the control of the thioredoxin system early during the evolution of streptophytes. We found no evidence for a GAPDH B in Chlamydomonas or other chlorophytes. Therefore, the evolution of a GAPDH B might represent a molecular characteristic (synapomorphy) of the streptophytes.

A total of 25 expressed genes encode components of the light-harvesting complex. There are some light-harvesting complex proteins, which Mesostigma shares only with the chlorophytes and red algae (e.g. so called fucoxanthin/ chlorophyll a-binding proteins). For others, we detected similar proteins only within embryophytes. However, the 


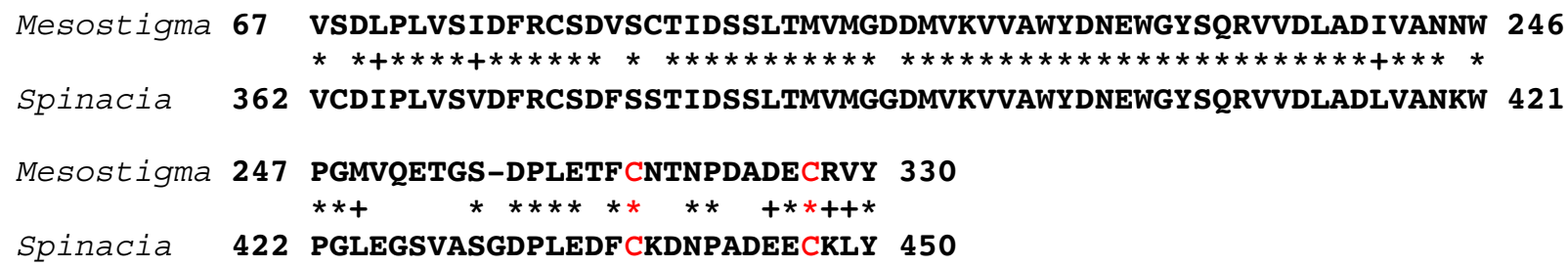

\section{Figure 3}

Alignment of the deduced amino acid sequence of the putative GAPDHB (Meso2a42g I 2) gene from Mesostigma with spinach (P I 2860) GAPDHB. The conserved cysteine residues are indicated in red letters. Numbers refer to the amino acid position (spinach) or nucleotide position (Mesostigma).

lhc proteins form a large superfamily and their phylogenetic analysis is beyond the scope of this study.

Several genes encode proteins of the photorespiratory C2cycle (glycolate phosphatase, peroxisomal glycolate oxidase, a component of the glycine decarboxylase enzyme complex, and a peroxisomal serine-glyoxylate transaminase). As in embryophytes, the NADH required for reduction of hydroxy pyruvate is produced by a peroxisomal NADH malate dehydrogenase.

A glycolate oxidase activity was never detected in chlorophytes by biochemical enzyme assays, but one Chlamydomonas protein is currently annotated as a glycolate oxidase (gene model C_340068, JGI Chlamydomonas reinhardtii v2.0) We therefore performed a phylogenetic analysis for glycolate oxidases and lactate dehydrogenases, which are both members of the same protein superfamily, from embryophytes, Mesostigma, Chlamydomonas, Cyanidioschyzon, Dictyostelium, a few metazoans and some bacteria (Fig. 4). The glycolate oxidases from embryophytes, Mesostigma and Cyanidioschyzon are monophyletic. In contrast, the glycolate oxidase-like sequence from Chlamydomonas clusters with bacterial sequences, which are annotated as lactate dehydrogenase and glycolate oxidases. Therefore, we conclude that, in agreements with the biochemical findings, Chlamydomonas does not contain a plant-type peroxisomal glycolate oxidase.

We did not find evidence for a hexokinase and sucrose biosynthesis in interphase cells of Mesostigma. Several ESTs represent plastidic pyruvate kinase, however, only a single EST coded for the cytosolic isoform. Expressed genes for PEP carboxylase and a cytosolic malate dehydrogenase are present, suggesting that malate may be the major substrate for respiration in the mitochondrion of
Mesostigma as in many embryophytes. The plastidic pyruvate kinase probably functions in the generation of acetylCoA required to sustain fatty acid synthesis in plastids.

Scales consist mainly of the 2-keto sugar acids 3-deoxymanno-octulosonic acid (2-keto-3-deoxy-oktonate, kdo), 5OMekdo, 3-deoxy-lyxo-heptulosaric acid, dha) and gal, galA, gul and some minor monosaccharides [25]. Expressed genes coding for kdo synthesis, and activation of kdo as CMP-kdo are present. The obtained sequence similar to a CMP-sialA transporter might actually be the CMP-kdo transporter necessary for uptake of CMP-kdo into the Golgi apparatus, as kdo and sialA are structural analogs. Interestingly, kdo-synthase and CMP-kdo-transferase are among the most conserved proteins between Mesostigma and the embryophytes. As in embryophytes [26], galA is synthesized via the UDP-glc dehydrogenase pathway and the myo-inositol oxygenase pathway. We could not detect the latter enzyme in Chlamydomonas or red algae.

Our EST-data support the presence of vitamin B12-biosynthesis and the production of a phosphagen phosphoarginine by arginine kinase in Mesostigma.

\section{Evolution of metabolism and cell structure}

259 expressed genes from Mesostigma showed similarity to proteins belonging to various metabolic pathways. A pairwise comparison of these genes with the genome of Chlamydomonas and the genomes and ESTs of the three embryophytes showed that Mesostigma shares more metabolic genes with the embryophytes than with Chlamydomonas, however, the overall $\mathrm{AI}$ is slightly higher with Chlamydomonas than with any embryophyte (AI, Table 6). Statistical analyses showed that the differences in AIs for the total metabolic enzyme data set are not significant (not shown). However, if we calculate AIs for dif- 


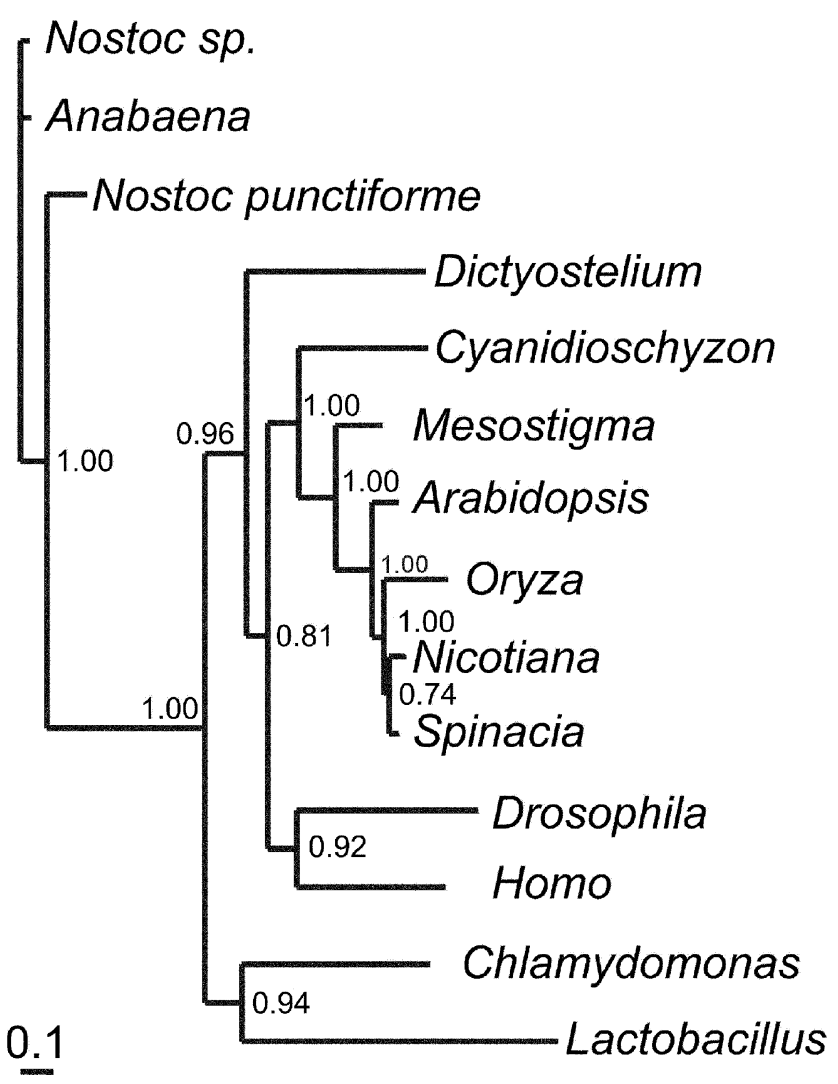

Figure 4

Phylogenetic tree of glycolate oxidase and glycolate oxidase-like genes. The tree shown was derived by Bayesian inference analysis from 402 amino acid positions using a mixed model for amino acid substitutions and a gamma correction for rate variation among sites. The Bayesian inference utilized MRBAYES, Ver. $3.0 *$ with posterior probabilities derived from 100000 generations and discarding a burnin of 1000. The tree obtained with a parsimony analysis using PHYLIP gave essentially the same topology.

ferent functional categories separately, we see that metabolic enzymes of the chloroplasts and mitochondria (photosynthesis except the Calvin cycle enzymes, fatty acid synthesis, synthesis of some amino acids, citric acid cycle, and respiration) were generally more conserved between Mesostigma and Chlamydomonas than between Mesostigma and the embryophytes (Table 6). In contrast, proteins of cytosolic pathways (nucleotide metabolism, NDP-sugar metabolism, and glycolysis) in Mesostigma were more similar to embryophyte proteins (Table 6),

Genes coding for information storage and processing, and cellular processes and signaling (Table 3) were overall more conserved between Mesostigma and the embryophytes than between Mesostigma and Chlamydomonas. Exceptions to this rule are proteins of the cytoskeleton (Table 7) and proteins involved in protein folding (chap- erones, Table 7) and plastidic proteases (not shown), which show higher AI values with Chlamydomonas than with the embryophytes. If the cytoskeletal proteins are removed from the data set, the differences between Mesostigma/Chlamydomonas genome and Mesostigma/embryophytes are statistical significant $(\mathrm{p}=0.000109$ for Mesostigma/Chlamydomonas versus Mesostigma/Physcomitrella; $\mathrm{p}=0.000703$ for Mesostigma/Chlamydomonas versus Mesostigma/Arabidopsis, $\mathrm{p}=0.006937$ for Mesostigma/Chlamydomonas versus Mesostigma/Oryza). Remarkably, the three embryophytes behave differently in our analysis. We obtained higher AI values with Physcomitrella regarding the categories protein folding (chaperones), vesicular transport, transcription, and regulation (Table 7). In contrast, proteins related to DNA structure, replication, cell cycle and RNA-metabolism were more conserved between Mesostigma and the angiosperms Arabidopsis and Oryza than between Mesostigma and Physcomitrella (Table 7).

\section{Discussion}

In this study, we have analyzed about 3000 expressed genes from the scaly green flagellate Mesostigma viride. We compared the expressed genes with the complete genomes from the angiosperms Arabidopsis thaliana and Oryza sativa, the chlorophyte Chlamydomonas reinhardtii, the red alga Cyanidioschyzon merolae and the diatom Thalassiosira pseudonana, as well as the ESTs from the moss Physcomitrella patens, and the red alga Porphyra yezoensis. Altogether, the Mesostigma proteome is more similar to the embryophytes than to Chlamydomonas, although Mesostigma and Chlamydomonas are both flagellate unicells. Mesostigma shares more genes with the embryophytes than with Chlamydomonas, including several enzymes confined to the streptophytes (e.g. GAPDH B, [Cu-Zn] superoxide dismutase), and the average identity of shared proteins is higher between Mesostigma and the embryophytes than between Mesostigma and Chlamydomonas. Therefore, we consider Mesostigma to be a member of the streptophytes, although Mesostigma clearly shares some ancestral characters with chlorophytes. Plastidic (with the exception of the Calvin cycle) and mitochondrial functions e.g. seem to be more conserved between Mesostigma and chlorophytes than between Mesostigma and embryophytes, i.e. these functions are more derived in embryophytes, probably due to adaptation of embryophytes to the terrestrial habitat. In contrast, other cellular functions except for the cytoskeleton are more conserved between Mesostigma and embryophytes than between Mesostigma and Chlamydomonas. Interestingly, in previous phylogenetic analyses plastidic and mitochondrial genes failed to show a clear relationship between Mesostigma and the streptophytes $[14,15]$, whereas actin and nuclear-encoded SSU rDNA phylogenies support the notion that Mesostigma is a member of the streptophytes [10-12]. The dif- 
Table 6: Comparison of Mesostigma genes related to metabolic functions with Chlamydomonas and three embryophytes. The average identity (AI) of pair-wise comparisons of Mesostigma expressed genes coding for the indicated metabolic function with the ESTs or genome of the given organisms are presented.

\begin{tabular}{|c|c|c|c|c|}
\hline Function 1 & Chlamydomonas & Physcomitrella & Arabidopsis & Oryza \\
\hline Metabolism (259) & 0.613 & 0.601 & 0.593 & 0.594 \\
\hline Plastidic metabolism $(107)^{2}$ & 0,595 & 0,572 & 0,567 & 0,572 \\
\hline Mitochondrial metabolism $(16)^{2}$ & 0,647 & 0,593 & 0,569 & 0,578 \\
\hline cytosolic metabolism (glycolysis, NDP-sugar metabolism, nucleotide synthesis, 22)2 & 0,568 & 0,639 & 0,641 & 0,632 \\
\hline
\end{tabular}

I Numbers in brackets indicate the number of genes in this category.

ferent evolutionary rates for different cellular functions observed in this study might explain this discrepancy.

We calculated the average identity (AI) values from automatically generated BLAST output alignments. Automatically derived alignments are prone to errors. However, we believe that our approach is justified for the following reasons: (1) the BLAST alignments cover only the conserved parts of proteins and our calculated AI values indicate that in most alignments more than half of the amino acids are identical enhancing the quality of the automatically produced alignments; (2) although small mistakes may occur, they are insignificant given the high number of amino acids used to calculate the AI. On average the BLAST alignments contained about 150 amino acids and therefore about 45,000 amino acid positions were used in the constrained data set. In large data sets small unbiased errors become irrelevant [27]. Our results indicate that at least 100 (better are 150-200) expressed genes have to be used to obtain statistically significant results. It could be argued that our analysis uses only similarity values and no real evolutionary distances. AI values can be easily converted into evolutionary distances using an approximation given by Kimura [28], with the effect that the differences between the various organisms become larger but no changes occur in the order of relatedness (included in Table 4). We conclude that the AI of proteins shared between different organisms represents a reasonable measure of evolutionary relatedness, if sufficiently large data sets are used.

In the following, we briefly discuss some major differences in coding potential observed between the different photosynthetic eukaryotic organisms.

11 of 18 proteins included in supplemental Table 1 [see Additional file 1] which are shared only by Mesostigma and Chlamydomonas are associated with flagellar functions such as axonemal dyneins or components of the IFT (intra-flagellar transport) machinery. Most likely, the angiosperms lost these proteins during evolution together with the ability to produce flagellate cells. The absence of these proteins in the ESTs from the moss Physcomitrella, is presumably due to the fact that ESTs from developing spermatozoids are not available.

Proteins shared by Mesostigma and the embryophytes but not present in chlorophytes perform diverse functions. There are some well known biochemical differences between chlorophytes and streptophytes such as the presence of $(\mathrm{Cu}-\mathrm{Zn})$ superoxide dismutase $[29,30]$ and glycolate oxidase in streptophytes [31,32] but not in chlorophytes. In addition, streptophytes use the DXP and mevalonate pathways for isoprene biosynthesis whereas chlorophytes posses only the DXP pathway [33]. For all these functions, we find molecular support in our expressed gene data set except for the mevalonate pathway of isoprene biosynthesis. Two genes matched two different enzymes of the DXP pathway; however, no matches for the MVA pathway were obtained, although the presence of this pathway has been demonstrated biochemically [33]. This could be due to the selective expression of one or the other pathway under different environmental conditions.

Remarkably, our list of proteins uniquely shared by Mesostigma and the embryophytes includes several proteins involved in steroid biosynthesis (e.g. a 3-oxo-5-beta-steroid dehydrogenase and a C-4 sterol oxidase), a homeobox protein of the knox family and proteins of the F-box family. The latter protein family underwent a dramatic expansion in the embryophytes (Arabidopsis has more than 700 members of this family).

Our expressed protein data set contains sequences similar to a protein involved in vitamin B-12 metabolism (present in rhodophytes and chlorophytes), an arginine kinase and a ARL6 protein, the latter two are absent in chlorophytes, embryophytes and red algae. It has been shown that arginine kinase is part of the ATP regeneration system in cilia of Paramecium [34]. Chlamydomonas lacks arginine kinase and recently Pazour et al. [35] showed that enzymes of the late glycolytic pathway are present in the flagella of Chlamydomonas, suggesting that the ATP required for flagellar function is produced by the glycolytic pathway in Chlamydomonas. The ARL6 protein has 
Table 7: Comparison of Mesostigma genes related to cell structure functions with the genome or ESTs of Chlamydomonas and three embryophytes. The average identity of pair-wise comparisons of Mesostigma expressed genes coding for the indicated cellular functions with the ESTs or genomes of the given organisms are presented.

\begin{tabular}{|c|c|c|c|c|}
\hline Function 1 & Chlamydomonas & Physcomitrella & Arabidopsis & Oryza \\
\hline Cell structure $(20 \mathrm{I})$ & 0.629 & 0.641 & 0.627 & 0.618 \\
\hline Cytoskeleton (7) & 0.751 & 0.729 & 0.0 .731 & 0.731 \\
\hline Protein folding/Chaperones (2I) & 0.685 & 0.605 & 0.580 & 0.581 \\
\hline Cytosolic protein degradation (22) & 0.682 & 0.733 & 0.722 & 0.703 \\
\hline Vesicular transport (22) & 0.633 & 0.701 & 0.680 & 0.673 \\
\hline Regulation (18) & 0.585 & 0.599 & 0.548 & 0.544 \\
\hline DNA structure, replication, cell cycle (2I) & 0.580 & 0.616 & 0.655 & 0.625 \\
\hline Transcription (16) & 0.684 & 0.675 & 0.647 & 0.623 \\
\hline RNA metabolism (23) & 0.599 & 0.619 & 0.637 & 0.637 \\
\hline
\end{tabular}

I Numbers in brackets indicate the number of genes in this category.

been implicated in protein translocation at the rER [36], although its exact function is still not known.

There are some typical embryophyte pathways that we failed to detect in Mesostigma, e.g. sucrose metabolism, hexokinase, and enzymes of cellulose biosynthesis. There are no reports about the presence of sucrose metabolism and hexokinase in green algae in the literature, whereas embryophyte-like Ces genes (catalytical subunit of cellulose synthase) have been reported in the streptophyte alga Mesotaenium [37]. Although we cannot exclude that Mesostigma lost these genes, we do expect to find theses genes in the genome of Mesostigma.

\section{Evolution of photosynthesis and photorespiration}

It is well known that embryophytes and chlorophytes differ in important aspects of photosynthesis and its regulation, and in photorespiration (e.g., presence of GAPDHB, number of enzymes regulated by thioredoxin, glycolate oxidase vs. glycolate dehydrogenase, and presence or absence of $(\mathrm{Cu}-\mathrm{Zn})$ superoxide dismutase $)$.

Table 8 summarizes the available information on the regulation of plastidic proteins by the thioredoxin system. The number of thioredoxin-regulated proteins has apparently increased during evolution and Mesostigma in this respect most closely resembles the embryophytes. Similarly, the peroxisomes of Mesostigma have been biochemically characterized as "leaf-type peroxisomes" [38] in full agreement with our EST-data. In contrast, chlorophytes lack glycolate oxidase and photorespiration involves only chloroplast and mitochondrial enzymes [38]. Interestingly, red algae possess a peroxisomal glycolate oxidase whereas the other enzymes of the photorespiratory cycle are located in the mitochondrion [32]. Thus, it seems likely that at the onset of streptophyte evolution major changes occurred in the regulation of the Calvin cycle and the subcellular organization of photorespiration. What might have been the driving force for these changes? We note that rhodophytes and chlorophytes both presumably evolved in a marine environment [red algae in a coastal benthic habitat, whereas chlorophytes proliferated as marine phytoplankton [39]]. Streptophyte algae most likely originated in a freshwater/brackish environment. In contrast to their marine counterparts, they had to deal with much higher light intensities and fluctuating environmental conditions such as salinity and temperature. With higher temperature, the rate of photorespiration increases. The observed changes in regulation of the Calvin cycle and photorespiration might be adaptations to this stress. It is possible that these adaptations to a shallow freshwater/brackish environment prepared streptophytes to colonize the terrestrial habitat later during evolution. In this respect we note that in extant chlorophytes activation of carbon concentrating mechanisms (CCM) is the dominant reaction to compensate for increased photorespiratory losses [38]. In contrast, streptophytes are able to channel large amounts of glycolate through the photorespiratory cycle [38]. According to Badger and Price [40] CCMs did not evolve until 400 million years ago, long after streptophytes had evolved and the colonization of the terrestrial habitat by streptophyte algae took place. Therefore during the palaeozoic era with reduced $\mathrm{CO}_{2}$ - and increased $\mathrm{O}_{2}$-levels [40] streptophyte algae might have had an advantage over chlorophyte algae allowing them to colonize the terrestrial habitat during that time.

\section{Conclusion}

In summary, our EST analysis shows that Mesostigma shares more genes with the embryophytes than with the chlorophyte Chlamydomonas reinhardtii, although both organisms are flagellate unicells. Thus, it seems likely that many typical biochemical characteristics of streptophytes evolved early during the evolution of streptophytes, i.e. before the transition to land. Alternatively, such characteristics may haven been lost in the chlorophyte lineage or remain to be discovered in other chlorophytes. A decision 
Table 8: Regulation of plastidic enzymes by the thioredoxin system. Proteins similar to embryophyte plastidic thioredoxin-regulated proteins were identified in the genomes of Cyanidioschyzon, Chlamydomonas, and the ESTs of Mesostigma using the BLASTP or BLASTX algorithms. A putative thioredoxin-regulated orthologue as revealed by the conserved cysteine residues is indicated with +. An asterisk indicates putative cyanobacterial/plastidic proteins, which do not contain the conserved cysteines required for thioredoxinregulation. Missing enzymes are indicated with -.

\begin{tabular}{lccccc}
\hline & Cyanobacteria & $\begin{array}{c}\text { Rhodophytes Cya- } \\
\text { nidioschyzon }\end{array}$ & $\begin{array}{c}\text { Chlorophytes } \\
\text { Chlamydomonas }\end{array}$ & Mesostigma & Embryophytes \\
\hline PRK & + & + & + & + & + \\
SDPase & $*$ & + & + & + & + \\
G6PDH & $*$ & $* 1)$ & + & + & + \\
FBPase & $*$ & $*$ & - & + & + \\
$\gamma$-ATPase & $*$ & - & $+2)$ & + & + \\
GABDHB & - & - & $*$ & + & + \\
NADP-MDH & - & - & + & + \\
Rubisco activase & $-3)$ & & $\left.+)^{4}\right)$ & +
\end{tabular}

n.d. not detected in Mesostigma. I) In Galdieria (Cyanidioschyzon) 2 (I) of the 3 conserved cysteines occurring in the Viridiplantae are present [48]. 2) Chlorophyte NADP-malate dehydrogenase possesses a C- and N-terminal extension like the embryophyte enzyme, however only the C-terminal cysteines of the embryophyte enzyme are conserved $[49,50]$. 3) A few cyanobacteria contain an unusual rubisco activase. Only the central AAA ${ }^{+}$ domain shows similarity to plant rubisco activases, whereas the $\mathrm{N}$ and $\mathrm{C}$ terminal domain are very different [5I]. ${ }^{4}$ Many angiosperms contain two forms of rubisco activase. Only the long form is regulated by the thioredoxin system [52].

between these alternatives requires further information on the genomes of other preferentially early branching chlorophytes such as Pyramimonas.

Our EST-analysis of Mesostigma supports the notion that this organism should be a suitable unicellular model for the last flagellate common ancestor of the streptophytes.

\section{Methods}

\section{Plant material, RNA preparation and construction of} libraries

Total RNA was isolated from cultures of Mesostigma viride Lauterborn (strain NIES 476, Tsukuba, Japan) and mRNA isolated using the mTRAP ${ }^{\mathrm{rm}}$ Total Kit (Active Motif). $5 \mu \mathrm{g}$ of mRNA were converted into cDNA using the SuperScript $^{\mathrm{TM}}$ Plasmid System (Invitrogen) and the cDNA obtained was fractionated by column chromatography. A large and a small size fraction were cloned into the pSPORT1 vector (Invitrogen).

Normalized full-length cDNA was prepared by Evrogen JSC (Moscow, Russia). cDNA was prepared from total RNA using the SMART approach [41] normalized using the DSN normalization method [42] and then amplified by PCR. cDNAs were either directly cloned into a pPCRScript Amp SK(+) Vector (PCR-Script Amp Cloning Kit, Stratagene) or a large size fraction was isolated by column chromatography and then cloned into a pGEM-T Easy vector (Promega). All libraries were transformed into TOP 10 E. coli cells (Invitrogen) by electroporation.

\section{Sequencing, contig assembly and data analysis} Clone preparation and sequencing Isolated plasmids were sequenced by the cycle sequencing method using an ABI3700 96 capillary sequencer. A min- imal contig set was assembled using the phrap assembler and all contigs were manually curated.

\section{Annotation}

Each contig was compared as 3-frame translations to the protein databases Swissprot and genpept using blastx. Furthermore, all contigs were compared using the tBLASTX search algorithm to the genome sequence of Chlamydomonas reinhardtii, Cyanidioschyzon merolae, Thalassiosira pseudonana, Arabidopsis thaliana, Oryza sativa and to the EST databases of Physcomitrella patens and Porphyra yezoensis. The results were compiled to an Excel compatible file. Analyses of COG and KOG categories $[43,44]$ and Interpro protein domains [45] for the contigs were also performed.

\section{Analysis of metabolic pathways}

Using the metabolic pathways present at the AraCyc website [46], we identified all expressed genes with significant similarity to Arabidopsis genes present in AraCyc. Expressed genes that showed no significant similarity to Arabidopsis genes but to enzymes from other organisms were assigned to a pathway using the MetaCyc database [47].

\section{Phylogenetic analysis of glycolate oxidase}

Thirteen glycolate oxidase/lactate dehydrogenase sequences were obtained from public databases (Cyanidioschyzon merolae [KEGG:CMQ436C]; Chlamydomonas reinhardtii [JGI:C_340068]; Spinacia oleracea [SwissProt:P05414]; Nostoc punctiforme PCC 73102 [Genbank:ZP 00106740.1]; Nostoc sp. PCC 7120 [Genbank:BAB77694.1]; Anabaena variabilis ATCC 29413 [Genbank:ZP_00160276.2]; Arabidopsis thaliana [Genbank:CAB78838], Oryza sativa [Genbank:AAB82143], 
Nicotiana tabacum [Genbank:AAC33509], Homo sapiens [Genbank:CAC34364], Drosophila melanogaster [Genbank:AAO41411], Dictyostelium discoideum [Genbank:XP_629946], Lactobacillus johnsonii NCC 533 [Genbank:NP 965805]). The nearly complete Mesostigma glycolate oxidase sequence was obtained by complete sequencing of EST clone Meso2b12b08. The sequences were aligned using Clustal X. The alignment was checked manually. Phylogenetic analyses were performed using the Phylip (neighbour joining and parsimony method) and MRBAYES software v 3.0 (Bayesian inference).

\section{Data deposition}

Sequence data from this article have been deposited with the EMBL/Genbank data libraries under accession numbers DN254242 to DN264595.

\section{Authors' contributions}

AS constructed the libraries and participated in the analysis of the EST-data. GG sequenced the ESTs, participated in the analysis of the EST-data and helped to draft the manuscript. MF participated in the analysis of the EST-data. MM participated in the design of the study, the analysis of the EST-data and helped to draft the manuscript. BB conceived the study, and participated in its design, coordination and analysis of the EST-data, and helped to draft the manuscript. All authors read and approved the final manuscript.

\section{Additional material}

\author{
Additional file 1 \\ Supplemental Table 1 \\ Click here for file \\ [http://www.biomedcentral.com/content/supplementary/1471- \\ 2229-6-2-S1.doc]
}

\section{Additional file 2}

Supplemental Table 2

Click here for file

[http://www.biomedcentral.com/content/supplementary/14712229-6-2-S2.doc]

\section{Acknowledgements}

This work was supported by the DFG (Bel779/7-I).

\section{References}

I. Sanderson MJ, Thorne JL, Wikstrom N, Bremer K: Molecular evidence on plant divergence times. Am J Bot 2004, 91:1656-1665.

2. Bateman RM, Crane PR, DiMichele WA, Kenrick PR, Rowe NP, Speck T, Stein WE: Early evolution of land plants: Phylogeny, physiology, and ecology of the primary terrestrial radiation. Annu Rev Ecol Syst 1998, 29:263-292.

3. Kenrick P, Crane PR: The origin and early diversification of land plants Washington, London: Smithsonian Institution Press; 1997.

4. Graham LE: Origin of Land Plants New York: John Wiley \& Sons, Inc; 1993.
5. Waters ER: Molecular adaptation and the origin of land plants. Molecular Phylogenetics and Evolution 2003, 29:456-463.

6. Bremer K: Summary of green plant phylogeny and classification. Cladistics 1985, I:369-385.

7. Mattox KR, Stewart KD: Classification of green algae: $\mathbf{A}$ concept based on comparative cytology. In Systematics of the green algae Edited by: Irvine DEG, John DM. London: Academic Press; 1984:29-72.

8. Nakayama T, Marin B, Kranz HD, Surek B, Huss VAR, Inouye I, Melkonian $M$ : The basal position of scaly green flagellates among the green algae (Chlorophyta) is revealed by analyses of nuclear-encoded SSU rRNA sequences. Protist 1998, I 49:367-380.

9. Huss VAR, Kranz HD: Charophyte evolution and the origin of land plants. Plant Syst Evol 1997:103-II4.

10. Karol KG, McCourt RM, Cimino MT, Delwiche CF: The closest living relatives of land plants. Science 200I, 294:235I-2353.

II. Bhattacharya D, Weber K, An SS, Berning-Koch W: Actin phylogeny identifies Mesostigma viride as a flagellate ancestor of the land plants. J Mol Evol 1998, 47:544-550.

12. Marin B, Melkonian M: Mesostigmatophyceae, a new class of streptophyte green algae revealed by SSU rRNA sequence comparisons. Protist 1999, 150:399-417.

13. Melkonian M, Marin B, Surek B: Phylogeny and Evolution of the Algae. In Biodiversity and Evolution Edited by: Arai R, Kato M, Doi Y. Tokyo: The National Science Museum Foundation; 1995:153-176.

14. Turmel M, Otis C, Lemieux C: The Complete Mitochondrial DNA Sequence of Mesostigma viride Identifies This Green Alga as the Earliest Green Plant Divergence and Predicts a Highly Compact Mitochondrial Genome in the Ancestor of All Green Plants. Mol Biol Evol 2002, 19:24-38.

15. Lemieux C, Otis C, Turmel M: Ancestral chloroplast genome in Mesostigma viride reveals an early branch of green plant evolution. Nature 2000, 403:649-652.

16. Delwiche CF, Karol KG, Cimino MT, Sytsma KJ: Phylogeny of the genus Coleochaete (Coleochaetales, Charophyta) and related taxa inferred by analysis of the chloroplast gene rbcL. J Phycol 2002, 38:394-403.

17. Poethig RS: Life with 25,000 genes. Genome Res 200 I, I I:313-316.

18. Palmer JD, Soltis DE, Chase MW: The plant tree of life: An overview and some points of view. Am J Bot 2004, 9 I: I $437-$ I 445.

19. Lewis LA, McCourt RM: Green algae and the origin of land plants. American Journal of Botany 2004, 9 I: 1535-1556.

20. McCourt RM, Delwiche CF, Karol KG: Charophyte algae and land plant origins. Trends Ecol Evol 2004, 19:661-666.

21. Graham LE, Cook ME, Busse JS: The origin of plants: Body plan changes contributing to a major evolutionary radiation. PNAS 2000, 97:4535-4540.

22. Quinn GP, Keough MJ: Experimental design and data analysis for biologists Cambridge: Cambridge University Press; 2002.

23. Ohlrogge J, Benning C: Unraveling plant metabolism by EST analysis. Curr Opin Plant Biol 2000, 3:224-228.

24. Cerff R, Chambers SE: Subunit Structure of Higher-Plant Glyceraldehyde-3-Phosphate Dehydrogenases (Ec I.2.1.12 and Ec 1.2.1.13). J Biol Chem 1979, 254:6094-6098.

25. Becker B, Becker D, Kamerling JP, Melkonian M: 2-keto-sugar acids in green flagellates: A chemical marker for prasinophycean scales. JPhycol I 99I, 27:498-504.

26. Kanter U, Usadel B, Guerineau F, Li Y, Pauly M, Tenhaken R: The inositol oxygenase gene family of Arabidopsis is involved in the biosynthesis of nucleotide sugar precursors for cell-wall matrix polysaccharides. Planta 2005, 22 I:243-254.

27. Delsuc F, Brinkmann H, Philippe H: Phylogenomics and the reconstruction of the tree of life. Nature Reviews Genetics 2005 , 6:36I-375.

28. Kimura M: The Neutral Theory of Molecular Evolution Cambridge: Cambridge University Press; 1983.

29. Fink RC, Scandalios JG: Molecular evolution and structure-function relationships of the superoxide dismutase gene families in angiosperms and their relationship to other eukaryotic and prokaryotic superoxide dismutases. Arch Biochem Biophys 2002, 399:19-36.

30. Dejesus MD, Tabatabai F, Chapman DJ: Taxonomic Distribution of Copper-Zinc Superoxide-Dismutase in Green-Algae and Its Phylogenetic Importance. J Phycol 1989, 25:767-772. 
31. Frederics E, Gruber PJ, Tolbert NE: Occurrence of Glycolate Dehydrogenase and Glycolate Oxidase in Green Plants Evolutionary Survey. Plant Physiol 1973, 52:3 I 8-323.

32. Igamberdiev AU, Lea PJ: The role of peroxisomes in the integration of metabolism and evolutionary diversity of photosynthetic organisms. Phytochemistry 2002, 60:65I-674.

33. Schwender J, Gemunden C, Lichtenthaler HK: Chlorophyta exclusively use the I-deoxyxylulose 5-phosphate/2- C-methylerythritol 4-phosphate pathway for the biosynthesis of isoprenoids. Planta 200I, 21 2:41 6-423.

34. Noguchi M, Sawadas T, Akazawa T: ATP-regenerating system in the cilia of Paramecium caudatum. Journal of Experimental Biology 200I, 204: 1063-107I.

35. Pazour GJ, Agrin N, Leszyk J, Witman GB: Proteomic analysis of a eukaryotic cilium. J Cell Biol 2005, I 70: 103-I I3.

36. Pasqualato $S$, Renault $L$, Cherfils J: Arf, Arl, Arp and Sar proteins: a family of GTP-binding proteins with a structural device for 'front-back' communication. EMBO Rep 2002, 3: I035-104I.

37. Roberts AW, Roberts E: Cellulose synthase (CesA) genes in algae and seedless plants. Cellulose 2004, I I:419-435.

38. Stabenau $\mathrm{H}$, Winkler U: Glycolate metabolism in green algae. Physiol Plant 2005, I 23:235-245.

39. Falkowski PG, Katz ME, Knoll AH, Quigg A, Raven JA, Schofield O, Taylor FJR: The evolution of modern eukaryotic phytoplankton. Science 2004, 305:354-360.

40. Badger MR, Price GD: CO2 concentrating mechanisms in cyanobacteria: molecular components, their diversity and evolution. J Exp Bot 2003, 54:609-622.

41. Zhu YY, Machleder EM, Chenchik A, Li R, Siebert PD: Reverse transcriptase template switching: a SMART approach for fulllength cDNA library construction. Biotechniques 200I, 30:892-897.

42. Shagin DA, Rebrikov DV, Kozhemyako VB, Altshuler IM, Shcheglov AS, Zhulidov PA, Bogdanova EA, Staroverov DB, Rasskazov VA, Lukyanov S: A novel method for SNP detection using a new duplex-specific nuclease from crab hepatopancreas. Genome Res 2002, I 2: 1935-1942.

43. Tatusov RL, Fedorova ND, Jackson JD, Jacobs AR, Kiryutin B, Koonin EV, Krylov DM, Mazumder R, Mekhedov SL, Nikolskaya AN, Rao BS, Smirnov S, Sverdlov AV, Vasudevan S, Wolf YI, Yin J], Natale DA: The COG database: an updated version includes eukaryotes. $B m c$ Bioinformatics 2003, 4:4I.

44. Tatusov RL, Galperin MY, Natale DA, Koonin EV: The COG database: a tool for genome-scale analysis of protein functions and evolution. Nucleic Acids Res 2000, 28:33-36.

45. Apweiler R, Attwood TK, Bairoch A, Bateman A, Birney E, Biswas M, Bucher P, Cerutti T, Corpet F, Croning MDR, Durbin R, Falquet L, Fleischmann W, Gouzy J, Hermjakob H, Hulo N, Jonassen I, Kahn D, Kanapin A, Karavidopoulou Y, Lopez R, Marx B, Mulder NJ, Oinn TM, Pagni M, Servant F, Sigrist C], Zdobnov EM: The InterPro database, an integrated documentation resource for protein families, domains and functional sites. Nucleic Acids Res 200I, 29:37-40.

46. [http://www.arabidopsis.org/tools/aracyc/]

47. [http://metacyc.org/META/server.html]

48. Ruelland E, Miginiac-Maslow M: Regulation of chloroplast enzyme activities by thioredoxins: activation or relief from inhibition? Trends Plant Sci 1999, 4:136-141.

49. Ocheretina O, Haferkamp I, Tellioglu H, Scheibe R: Light-modulated NADP-malate dehydrogenases from mossfern and green algae: insights into evolution of the enzyme's regulation. Gene 2000, 258: 147-I54.

50. Lemaire SD, Quesada A, Merchan F, Corral JM, Igeno MI, Keryer E, Issakidis-Bourguet E, Hirasawa M, Knaff DB, Miginiac-Maslow M: NADP-malate dehydrogenase from unicellular green alga Chlamydomonas reinhardtii. A first step toward redox regulation. Plant Physiol 2005, 137:5 I 4-521.

5I. Portis AR: Rubisco activase - Rubisco's catalytic chaperone. Photosynth Res 2003, 75: I I-27.

52. Schurmann P, Jacquot JP: Plant thioredoxin systems revisited. Annu Rev Plant Physiol Plant Molec Biol 2000, 5 I:37 I -400.
Publish with Bio Med Central and every scientist can read your work free of charge

"BioMed Central will be the most significant development for disseminating the results of biomedical research in our lifetime. "

Sir Paul Nurse, Cancer Research UK

Your research papers will be:

- available free of charge to the entire biomedical community

- peer reviewed and published immediately upon acceptance

- cited in PubMed and archived on PubMed Central

- yours - you keep the copyright

Submit your manuscript here:

http://www.biomedcentral.com/info/publishing_adv.asp
BioMedcentral 